\title{
Rosacea and Helicobacter pylori: links and risks
}

This article was published in the following Dove Press journal:

Clinical, Cosmetic and Investigational Dermatology

10 August 2017

Number of times this article has been viewed

\section{Elizabeth Lazaridou' Chrysovalantis Korfitis ${ }^{2}$ Christina Kemanetzi' Elena Sotiriou' \\ Zoe Apalla' \\ Efstratios Vakirlis' Christina Fotiadou' \\ Aimilios Lallas' Demetrios loannides'}

${ }^{1}$ First Department of Dermatology and Venereology, Aristotle University Medical School, Thessaloniki, Greece; ${ }^{2}$ Department of Dermatology, 40 I General Army Hospital, Athens, Greece
Correspondence: Elizabeth Lazaridou First Department of Dermatology and Venereology, Aristotle University Medical School, 124 Delphon Street, 54643 Thessaloniki, Greece

Tel +30 23I 0242433

Email bethlaz@med.auth.gr
Abstract: Rosacea is a chronic skin disease characterized by facial erythema and telangiectasia. Despite the fact that many hypotheses have been proposed, its etiology remains unknown. In the present review, the possible link and clinical significance of Helicobacter pylori in the pathogenesis of rosacea are being sought. A PubMed and Google Scholar search was performed using the terms "rosacea", "H.pylori", "gastrointestinal disorders and H.pylori", "microorganisms and rosacea", "pathogenesis and treatment of rosacea", and "risk factors of rosacea", and selected publications were studied and referenced in text. Although a possible pathogenetic link between $H$. pylori and rosacea is advocated by many authors, evidence is still interpreted differently by others. We conclude that further studies are needed in order to fully elucidate the pathogenesis of rosacea.

Keywords: eradication, Helicobacter pylori, pathogenesis, rosacea

\section{Introduction}

Rosacea is a chronic inflammatory skin disorder characterized by erythema and telangiectasia, affecting predominantly the central part of the face, accounting for almost $1 \%$ of all skin disorders diagnosed by dermatologists. ${ }^{1}$ It is more common in Northern and Western Europe. It has been nicknamed as the "curse of the Celts" although such claim has been disputed. ${ }^{2}$ Caucasians are more commonly affected by rosacea, ${ }^{3}$ which also concerns women between the ages 30 and 60 , almost three times as much as men. The diagnosis of rosacea is based on specific clinical features as there is no laboratory test to confirm the diagnosis. It is divided into four different subtypes, erythematotelangiectatic, papulopustular, phymatous, and ocular. Four stages of the disease have been described. First, frequent flushing; second, persistent facial erythema with telangiectasias and skin sensitivity; third, papules, pustules, with ocular changes (blepharoconjunctivitis, meibomitis, iritis, burning, itching, and photophobia); $;^{4-7}$ and fourth, tissue hyperplasia and rhinophyma. Besides cutaneous symptoms, some studies concluded that rosacea is a disorder with various upper gastrointestinal symptoms. ${ }^{8-10}$ The classification of rosacea is important because both the pathogenesis and the treatment correspond to the specific form of the disease. It is likely that distinct subtypes respond differently to treatment. The causes and pathophysiology of the disease are not yet clear, since it is a multifactorial disorder. ${ }^{11}$

In the present review, the search terms which were used were "rosacea", "H.pylori", "gastrointestinal disorders and H.pylori", "microorganisms and rosacea", "pathogenesis and treatment of rosacea", "risk factors of rosacea". Our research was based on 
peer-reviewed publications found through the PubMed and Google Scholar literature databases, as well as the Google search engine. The articles refer to clinical trials, cohort studies, reviews, and epidemiological studies. Both old and recent articles were selected in order to study the evolution in prevalence of the search terms. Even though the total number of papers is limited, an attempt to include both small-scale and larger studies was made. Reviews from both dermatological and gastroenterological sources were included, in order to achieve a more integrated approach.

\section{Pathogenesis of rosacea}

The factors implicated in the pathogenesis of rosacea are endocrinological, pharmacological, immunological, climatical, dietary habits, free radicals, ultraviolet radiation, microbial agents, ${ }^{4,12,13}$ inheritance, ${ }^{14}$ inherent cutaneous vascular abnormalities, ${ }^{4,15}$ pilosebaceous unit abnormalities, ${ }^{16}$ and matrix degeneration. ${ }^{17}$ The nosological subtypes of rosacea probably represent different response to a combination of these factors.

Flushing appears as an inappropriate response to hyperthermia. ${ }^{4,15}$ Chronic sun exposure is responsible for the destruction of cutaneous blood vessels and dermal connective tissue ${ }^{4,15,18,19}$ and as a consequence, elastotic granuloma is a common histological finding, linked to disease development. ${ }^{19}$ Matrix degeneration has also been suggested as a possible factor. ${ }^{4}$ The pathophysiological association of rosacea with alimentary tract disorders is not clear. ${ }^{4}$ The association of alcohol consumption and caffeine intake with skin changes is not fully established even in the case of rhinophyma and alcohol. ${ }^{20}$ The inheritance of rosacea has not been thoroughly investigated. GSTM1 and GSTT1 null genotypes have been shown to be associated with an increased risk of rosacea and BsmI polymorphism of the $V D R$ gene has been correlated with fulminant rosacea. ${ }^{14,21}$ Abram et $\mathrm{al}^{20}$ concluded that the strongest risk factor in their study was the presence of family history. In the same study, the risk of developing rosacea was higher among ex-smokers than among current smokers or lifelong non-smokers, being in dissonance with previous studies. $^{22}$

Microorganisms have been addressed in a variety of studies as pathogenic factors. Mite-related bacteria, ${ }^{23}$ staphylococcus epidermidis, ${ }^{24}$ chlamydia pneumonia, ${ }^{25}$ bacterial toxins, ${ }^{24}$ and antimicrobial peptides ${ }^{26-28}$ have been addressed as potential trigger agents for rosacea. Demodex folliculorum, the small intestinal bacterial overgrowth (SIBO) and Helicobacter pylori are the most investigated, studied but also debated regarding their contribution in the pathogenesis of rosacea.
D. folliculorum is a mite, found almost in everyone ${ }^{4}$ but its density is higher in rosacea patients ${ }^{18,19}$ mainly in the papulopustular type. ${ }^{4}$ Five mites per follicle or five per $\mathrm{cm}^{2}$ are thought to be pathogenic. ${ }^{29}$ However, the dissenting opinion alleges that the fact that the prevalence of Demodex reaches almost $100 \%$ in healthy adults does not imply an etiologic relation to rosacea. ${ }^{30,31}$

SIBO is defined as unexpected microbial concentration in jejunal aspirate culture. In the general population the prevalence of SIBO is estimated to be $2.5 \%-22 \%$, ${ }^{32}$ while in rosacea patients it reaches the rate of $46 \% .{ }^{33}$ The association between SIBO and rosacea is not well-established yet. There are some controversial studies on the subject. Parodi et al contend that eradication of SIBO has been associated with a significant improvement in skin. ${ }^{33}$ However, Gravina et al concluded that SIBO does not seem to play a role in rosacea. ${ }^{9}$

\section{Clinical significance of $H$. pylori in rosacea}

$H$. pylori are gram-negative bacteria which comprise a large number of different strains. The term "Helicobacter pylori species-complex" has been proposed, due to the genetic diversity of $H$. pylori. ${ }^{34}$ The genomes of several strains have been completely sequenced. ${ }^{35-39}$

The detection of $H$. pylori infection is accomplished with several methods. Blood antibody test, stool antigen test or carbon urea breath test - which is metabolized by the bacterium producing detectable carbon dioxide in the breath - are all available. ${ }^{40}$ Also urine ELISA test and endoscopic biopsy comprise further options.

Since its discovery, $H$. Pylori has been associated with many diseases, not only gastrointestinal but also hematological, cardiopulmonary, metabolic, neurological, and dermatological. As far as it concerns the skin, there are reports for its implication in the pathogenesis of chronic spontaneous urticaria, Sjögren's syndrome, and Henoch-Schonlein purpura. ${ }^{41}$

Many studies have been performed in order to evaluate the connection between $H$. pylori infection and rosacea, however there is still controversy around the matter. There is a lack of information regarding the genetic features and the biology of $H$. pylori. It is proposed that the bacterium produces specific cytotoxins which lead to the release of histamine, prostaglandins, leukotrienes, and cytokines. Inflammatory mediators from an altered innate immune response lead to higher levels of ROS, ${ }^{42-44}$ whereas in treated rosacea the aforementioned level is lower. ${ }^{43}$ However, there are no distinct histological features in rosacea patients positive for H. pylori infection. ${ }^{19}$ 
One basic subject of studies is whether $H$. pylori prevalence is higher in patients with rosacea compared to the general population. Powel et al, in 1992, found that anti- $H$. pylori antibody levels were higher in rosacea patients. ${ }^{45}$ This result was duplicated in 1995 by Rebora et al. ${ }^{46}$ The higher prevalence of $H$. pylori has also been shown by Szlachcic et al's study ${ }^{47}$ in which $67 \%$ of rosacea patients had CagA positive strains, while only $32 \%$ of patients with non-ulcer dyspepsia were positive in this strain. The same investigator in 2002 found that there was a higher prevalence of $H$. pylori in patients with rosacea. ${ }^{8}$ In 2003 Argenziano et al demonstrated that anti-CagA antibodies were present in $75 \%$ of patients suffering both from rosacea and gastrointestinal symptoms, ${ }^{48}$ and in $81 \%$ of patients with rosacea and dyspepsia the serum anti-Hp IgG antibodies were detected. In the same year a pilot study conducted by Diaz et $\mathrm{al}^{49}$ concluded that rosacea is closely related to $H$. pylori infection, especially in patients suffering from an inflammatory subtype of the disease. One year later, in 2004, another study by Baz et al found that antiHp IgM was higher and IgG was lower in rosacea patients compared to heathy controls. In the same study the malondialdehyde level was increased and the antioxidant potential level was decreased. The authors concluded that rosacea is an oxidative stress condition, with increased ROS, regardless of H. pylori infection. ${ }^{50}$ In 2006, Boixeda de Miquel et $\mathrm{al}^{51}$ studied the effect of $H$. pylori eradication therapy in rosacea patients. They detected $H$. pylori using $\mathrm{C}^{13}$-urea breath test, IgG antibodies using ELISA, as well as upper gastrointestinal endoscopy with biopsy. All the infected patients, even those with no gastrointestinal symptoms, were treated until successful eradication was obtained. In this study, H. pylori was found in $84.1 \%$, and $61.4 \%$ of patients who presented with digestive symptoms, which denotes the relation between rosacea and digestive problems which confirms the study results of Sharma et al. ${ }^{52}$ Three months after eradication most of the patients had a complete regression of gastrointestinal symptoms and total or partial improvement of rosacea signs. The evolution of the disorder was independent of gender. Patients with papulopustular rosacea had a better response than those with erythematous, which confirmed the results from previous studies. ${ }^{53-55}$ All of them found clinical improvement of rosacea after eradication of the bacteria. Another study supporting the link between $H$. pylori and rosacea is the one conducted by Dakovic et al. ${ }^{56}$ They surveyed the improvement of ocular rosacea after treating the infection in a small number (seven) of patients with concomitant clinical and serological $H$. pylori infection. In these case series the ocular signs and symptoms showed more amelioration than the cutaneous ones. All the participants suffered from the erythematotelangiectatic subtype. ${ }^{56} \mathrm{~A}$ study in $2012^{57}$ was performed in order to identify the role of $H$. pylori in patients with rosacea and dyspeptic symptoms. Three different types of the bacteria (cagA, iceA, vacA) were evaluated. It was stated that vacA genotype is more common in papulopustular rosacea with significant elevation of $\operatorname{cagA} / \mathrm{vac} A$ $\mathrm{s} 1 \mathrm{~m} 1$ positivity in this rosacea subtype. The gastric biopsy showed that gastric ulceration was more frequent in patients with papulopustular rosacea and the eradication therapy was especially effective in those patients. They concluded that H. pylori plays an important role in rosacea patients with concomitant dyspeptic problems, especially in the papulopustular subtype. Gravina et al, ${ }^{9}$ also, not only found that the prevalence of bacteria is higher in rosacea patients but also the eradication of it improves skin signs. They observed that only the successful eradication of $H$. pylori led to the total or partial regression of the skin lesions. Most of the patients with unsuccessful treatment of the infection did not show any improvement. Rosacea symptoms improved only when second-line therapy for $H$. pylori was given. The most notable amelioration of skin symptoms was observed in stages II and III of the disease. A recent clinical trial was performed by Saleh et al. ${ }^{58}$ The authors concluded that the eradication of $H$. pylori with standard protocol is effective for rosacea symptoms. The telangiectasias and phymatous signs did not improve significantly.

Some authors support the pathogenetic role of $H$. pylori in rosacea because of the bacteria's involvement in the oxidative stress. $H$. pylori-infected patients have been reported to exhibit increased ROS activity and decreased plasma antioxidant factors such as ascorbic acid ${ }^{59,60}$ However, Gürer et al, having studied the seroprevalence of $H$. pylori and nitric oxide (NO) in rosacea, ${ }^{61}$ proposed that NO, which is thought to be associated with $H$. pylori, plays no role in the inflammation process of the disease.

In spite of all previous studies, there are some authors who dispute the link between rosacea and H. pylori. In 1992 Schneider et al, ${ }^{62}$ and in 1999 Son et al, ${ }^{63}$ failed to establish a role of $H$. pylori in rosacea patients. Herr and $Y_{0 u}^{64}$ concluded that the relationship between rosacea and $H$. pylori may be a myth. They studied both the prevalence of $H$. pylori and the effect on rosacea after the eradication treatment. Even in papulopustular subtype they noticed no significant improvement of skin lesions compared to placebo group. Although Bonamigo et $\mathrm{a}^{65}$ did not find any difference in prevalence of H. pylori in rosacea patients, they marked that the interference of previous use of systemic antibiotics could lead to incorrect 
conclusions. Another study which supports that there is no strong pathogenic contribution of $H$. pylori in rosacea is the study of Gürer et al, ${ }^{61}$ in which there was a higher proportion of anti-Hp antibodies in rosacea patients, but the levels of NO serum were normal.

Taking into consideration the fact that rosacea is a multifactorial disease, Abram et al concomitantly evaluated the risk factors of the disease. ${ }^{20}$ In this study, H. pylori rate was lower in flushers and higher in the papulopustular subtype. As far as it concerns the prevalence of bacteria in reference to age, it was significantly higher in the group of over 50 years of age, but this difference disappeared after age adjustment. This effect explains that the temporary signs of rosacea are due to younger age but not due to the lower seroprevalence. Another remarkable point was that rosacea patients who had taken systematic antibiotics did not have any statistical difference in $H$. pylori seropositivity compared to those who had used only topical treatment. According to this study no association between rosacea and H. pylori was found.

A clinical and laboratory study of rosacea in Northern Greece took place in our department with the objective of assessing the pathogenetic factors. ${ }^{18}$ Among them $H$. pylori was estimated but anti-Hp antibodies were almost at the same level between patients and controls, even when they were divided into two different age groups. Another result was that gender had no contribution to the etiology of the disease. Finally in the patients with no previous use of antibiotics, a strong correlation with $H$. Pylori was detected. ${ }^{18}$ However, the extended use of antibiotics may weaken the antigenic effect of $H$. pylori without eradicating it completely.

Egeberg et al in $2017,{ }^{66}$ performed a nationwide cohort study in order to investigate the links between rosacea and other gastrointestinal disorders (celiac disease, Crohn's disease, ulcerative colitis, H. pylori infection, SIBO, and irritable bowel syndrome). They established the prevalence of the disorders using nationwide administrative registers. They observed an increased frequency of $H$. pylori in rosacea patients but the risk of new-onset infection was not increased in patients with rosacea signs. They concluded that the prevalence of these gastrointestinal diseases is higher in patients with rosacea, and they found significant association between rosacea and celiac disease, Crohn's disease, ulcerative colitis, and irritable bowel syndrome, but not H. pylori infection and SIBO. They finally concluded that gastrointestinal complaints in rosacea patients justify clinical suspicion of disease.

\section{Discussion}

A lot of research has been carried out regarding the correlation of $H$. pylori with other diseases and its pathogenetic role. H. pylori infection is a very common chronic disease in humans. It has been linked with a multitude of gastrointestinal and extraintestinal diseases. It is implicated in hematologic, cardiopulmonary, metabolic, neurologic, and dermatologic manifestations, ${ }^{41}$ with iron deficiency anemia and immune thrombocytopenic purpura being the most well-established. ${ }^{9}$ $H$. pylori infection has been associated with several conditions affecting the skin, such as chronic urticaria, atopic dermatitis, Henoch-Schonlein purpura, Sweet's syndrome, psoriasis, alopecia areata, aphthous stomatitis, and lichen planus. ${ }^{9}$ Many recent studies have sought to identify the link of $H$. pylori to rosacea. These studies reached controversial conclusions and as a result, its role still remains ambiguous. Some authors believe that $H$. pylori indeed plays a pathogenetic role in rosacea, and others contradict any implication or accept it only as an aggravating factor rather than an etiological one. ${ }^{61,63}$ Another question is whether the eradication of bacteria improves rosacea and whether there is any association between $H$. pylori and rosacea subtype, given that some authors have identified that, in papulopustular subtype of rosacea, the prevalence of $H$. pylori and dyspeptic symptoms are increased. In addition, there are many distinct strains with different virulence, which may explain the different associations of $H$. pylori in these studies. Rosacea is a multifactorial disease which further complicates the evaluation of each risk factor alone, and conceals whether each factor acts separately or synergistically with the others. This probably explains the discrepancy between the different studies. Additionally, rosacea itself is a disorder with four different subtypes, each of which could have a diverse pathogenetic pathway. Moreover, $H$. pylori has been evaluated with various methods, such as $\mathrm{C}^{13}$-urea breath test, $H$. pylori stool antigen test, serological test using ELISA IgG test kit, and gastrointestinal endoscopy with biopsy or microbiologic culture. The studies used different evaluation methods, so this could be one of the reasons for the discrepancy between them regarding the prevalence of $H$. pylori in rosacea patients. The most precise method is biopsy, but its accuracy depends on the location of the specimen, requiring samples from five different sites. Furthermore, it is not very explicit whether $H$. pylori is the only gastric microorganism which plays a role, alone or in combination with SIBO or other gut microbiota, in the pathogenesis of rosacea.

Another important parameter is the role of antibiotics. Their previous use in participants may affect the results. 
Some authors advocate that they act by eradicating $H$. pylori, whereas others highlight a non-specific direct benefit from the use of antibiotics as the cause of clinical improvement. The confusion is further enhanced by the fact that some of the antibiotics known for their anti-inflammatory properties in rosacea, such as tetracyclines, metronidazole or macrolides ${ }^{67}$ are also used to treat $H$. pylori infection. ${ }^{68}$ Moreover, different studies have used different statistical methods which could constitute a further explanation for the controversial conclusions, taking also into account that much evidence is derived from case series with a small number of participants.

\section{Conclusion}

Rosacea is a skin disease with an obscure and complicated pathogenesis. Many mechanisms have been described but its etiology remains an enigma. There is not sufficient evidence regarding how determinant the role of $H$. pylori is. According to some authors, rosacea is correlated with gastrointestinal disorders which justifies further clinical and laboratory examination. Based on the fact that the studies were not extensive, controlled studies are required. Additional research is mandatory in the field of the microbiology of H. pylori as there are subtypes with different levels of virulence. Further studies are warranted to delve into the multifactorial nature of rosacea in order to elucidate the role of each factor in the pathogenesis of the disease.

\section{Disclosure}

The authors report no conflicts of interest in this work.

\section{References}

1. Buechner SA. Rosacea: an update. Dermatology. 2005;210(2):100-108.

2. Wollina U, Verma SB. Rosacea and rhinophyma: not curse of the Celts but Indo Eurasians. J Cosmet Dermatol. 2009;8(3):234-235.

3. TüzünY, Wolf R, Kutlubay Z, Karakuş O, Engin B. Rosacea and rhinophyma. Clin Dermatol. 2014;32(1):35-46.

4. Crawford GH, Pelle MT, James WD. Rosacea: I. Etiology, pathogenesis, and subtype classification. J Am Acad Dermatol. 2004;51(3): 327-341.

5. Tanzi EL, Weinberg JM. The ocular manifestations of rosacea. Cutis. 2001;68(2):112-114.

6. Erzurum SA, Feder RS, Greenwald MJ. Acne rosacea with keratitis in childhood. Arch Ophthalmol. 1993;111(2):228-230.

7. Akpek EK, Merchant A, Pinar V, Foster CS. Ocular rosacea: patient characteristics and follow-up. Ophthalmology. 1997;104(11): 1863-1867.

8. Szlachcic A. The link between Helicobacter pylori infection and rosacea. J Eur Acad Dermatol Venereol. 2002;16(4):328-333.

9. Gravina A, Federico A, Ruocco E, et al. Helicobacter pylori infection but not small intestinal bacterial overgrowth may play a pathogenic role in rosacea. United European Gastroenterol J. 2015;3(1): $17-24$.

10. Marks R, Beard RJ, Clark ML, Kwok M, Robertson WB. Gastrointestinal observations in rosacea. Lancet. 1967;1(7493):739-743.
11. Marks R. The enigma of rosacea. J Dermatolog Treat. 2007;18(6): 326-328.

12. Brittenham GM. Disorders of iron metabolism: Iron deficiency and overload. In: Hoffman R, Benz EJ, Shattil SJ, Furie B, Cohen HJ, Silberstein LE, editors. Haematology: Basic Principles and Practice. New York: Churchill Livingston; 1995: 492.

13. Lazaridou E, Giannopoulou C, Fotiadou C, Vakirlis E, Trigoni A, Ioannides D. The potential role of microorganisms in the development of rosacea. J Dtsch Dermatol Ges. 2011;9(1):21-25.

14. Yazici AC, Tamer L, Ikizoglu G, Kaya TI, Api H, Yildirim H, Adiguzel A. GSTM1 and GSTT1 null genotypes as possible heritable factors of rosacea. Photodermatol Photoimmunol Photomed. 2006;22(4):208-210.

15. Wilkin JK. Rosacea. Pathophysiology and treatment. Arch Dermatol. 1994;130(3):359-362.

16. Marks R, Harcourt-Webster JN. Histopathology of rosacea. Arch Dermatol. 1969;100(6):683-691.

17. Gomaa AH, Yaar M, Eyada MM, Bhawan J. Lymphangiogenesis and angiogenesis in non-phymatous rosacea. J Cutan Pathol. 2007;34(10):748-753.

18. Lazaridou E, Apalla Z, Sotiraki S, Ziakas NG, Fotiadou C, Ioannides D. Clinical and laboratory study of rosacea in northern Greece. $J$ Eur Acad Dermatol Venereol. 2010;24(4):410-414.

19. Aroni K, Tsagroni E, Lazaris AC, Patsouris E, Agapitos E. Rosacea: a clinicopathological approach. Dermatology. 2004;209(3):177-182.

20. Abram K, Silm H, Maaroos HI, Oona M. Risk factors associated with rosacea. J Eur Acad Dermatol Venereol. 2010;24(5):565-571.

21. Jansen T, Krug S, Kind P, Plewig G, Messer G. BsmI polymorphism of the vitamin D receptor gene in patients with the fulminant course of rosacea conglobata (rosacea fulminans). J Dermatol. 2004;31(3): 244-246.

22. Mills CM, Marks R. Environmental factors influencing rosacea. Clin Exp Dermatol. 1996;21(2):172-173.

23. Borgo SN, Sattler EC, Hogardt M, Adler K, Plewig G. PCR analysis for Wolbachia in human and canine Demodex mites. Arch Dermatol Res. 2009;301(10):747-752.

24. Dahl MV, Ross AJ, Schlievert PM. Temperature regulates bacterial protein production: possible role in rosacea. $\mathrm{J}$ Am Acad Dermatol. 2004;50(2):266-272.

25. Fernandez-Obregon A, Patton DL. The role of Chlamydia pneumoniae in the etiology of acne rosacea: response to the use of oral azithromycin. Cutis. 2007;79(2):163-167.

26. Yamasaki K, Di Nardo A, Bardan A, et al. Increased serine protease activity and cathelicidin promotes skin inflammation in rosacea. Nat Med. 2007;13(8):975-980.

27. Meyer-Hoffert U. Reddish, scaly, and itchy: how proteases and their inhibitors contribute to inflammatory skin diseases. Arch Immunol Ther Exp (Warsz). 2009;57(5):345-354.

28. Schauber J, Gallo RL. Antimicrobial peptides and the skin immune defense system. JAllergy Clin Immunol. 2009;124(3 Suppl 2):R13-18.

29. Hsu CK, Hsu MM, Lee JY. Demodicosis: a clinicopathological study. J Am Acad Dermatol. 2009;60(3):453-462.

30. Bonnar E, Eustace P, Powell FC. The Demodex mite population in rosacea. J Am Acad Dermatol. 1993;28(3):443-448.

31. Dubois S. Recherche du Demodex folliculorum hominis dans la peau saine [Research of Demodex folliculorum hominis in healthy skin]. Ann Dermatol Syph. 1910;1:188-190. French.

32. Bures J, Cyrany J, Kohoutova D, et al. Small intestinal bacterial overgrowth syndrome. World J Gastroenterol. 2010;16(24):2978-2990.

33. Parodi A, Paolino S, Greco A, et al. Small intestinal bacterial overgrowth in rosacea: clinical effectiveness of its eradication. Clin Gastroenterol Hepatol. 2008;6(7):759-764.

34. Hazell SL, Andrews RH, Mitchell HM, Daskalopoulous G. Genetic relationship among isolates of Helicobacter pylori: evidence for the existence of a Helicobacter pylori species-complex. FEMS Microbiol Lett. 1997;150(1):27-32.

35. Tomb JF, White O, Kerlavage AR, et al. The complete genome sequence of the gastric pathogen Helicobacter pylori. Nature. 1997;388(6642): $539-547$. 
36. Baltrus DA, Amieva MR, Covacci A, et al. The complete genome sequence of Helicobacter pylori strain G27. J Bacteriol. 2009;191(1):447-448.

37. Behrens W, Bönig T, Suerbaum S, Josenhans C. Genome sequence of Helicobacter pylori hpEurope strain N6. J Bacteriol. 2012;194(14): 3725-3726.

38. Mucito-Varela E, Castillo-Rojas G, Cevallos MA, et al. Complete genome sequence of Helicobacter pylori strain $29 \mathrm{CaP}$ isolated from a Mexican patient with gastric cancer. Genome Announc. 2016;4(1):e01512-01515.

39. Oh JD, Kling-Bäckhed H, Giannakis M, et al. The complete genome sequence of a chronic atrophic gastritis Helicobacter pylori strain: evolution during disease progression. Proc Natl Acad Sci U S A. 2006;103(26):9999-10004.

40. Stenström B, Mendis A, Marshall B. Helicobacter pylori--the latest in diagnosis and treatment. Aust Fam Physician. 2008;37(8):608-612.

41. Wong F, Rayner-Hartley E, Byrne MF. Extraintestinal manifestations of Helicobacter pylori: a concise review. World J Gastroenterol. 2014;20(34):11950-11961.

42. Yamasaki K, Gallo RL. The molecular pathology of rosacea. J Dermatol Sci. 2009;55(2):77-81.

43. Bakar O, Demirçay Z, Yuksel M, Haklar G, Sanisoglu Y. The effect of azithromycin on reactive oxygen species in rosacea. Clin Exp Dermatol. 2007;32(2):197-200.

44. Peus D, Vasa RA, Beyerle A, Meves A, Krautmacher C, Pittelkow MR. UVB activates ERK1/2 and p38 signaling pathways via reactive oxygen species in cultured keratinocytes. $J$ Invest Dermatol. 1999;112(5):751-756

45. Powell FC, Dawa MA, Duguid C. Positive Helicobacter pylori serology in rosacea patients. Ir J Med Sci. 1992;161:S75.

46. Rebora A, Drago F, Parodi A. May Helicobacter pylori be important for dermatologists? Dermatology. 1995;191(1):6-8.

47. Szlachcic A, Sliwowski Z, Karczewska E, Bielanski W, Pytko-Polonczyk J, Konturek SJ. Helicobacter pylori and its eradication in rosacea. $J$ Physiol Pharmacol. 1999;50(5):777-786.

48. Argenziano G, Donnarumma G, Iovene MR, Arnese P, Baldassarre MA, Baroni A. Incidence of anti-Helicobacter pylori and anti-CagA antibodies in rosacea patients. Int J Dermatol. 2003;42(8):601-604.

49. Diaz C, O'Callaghan CJ, Khan A, Ilchysyn A. Rosacea: a cutaneous marker of Helicobacter pylori infection? Results of a pilot study. Acta Derm Venereol. 2003;83(4):282-286.

50. Baz K, Cimen MY, Kokturk A, et al. Plasma reactive oxygen species activity and antioxidant potential levels in rosacea patients: correlation with seropositivity to Helicobacter pylori. Int $J$ Dermatol. 2004;43(7):494-497.

51. Boixeda de Miquel D, Vazquez Romero M, Vazquez Sequeiros E, et al. Effect of helicobacter pylori eradication therapy in rosacea patients. Rev Esp Enferm Dig. 2006;98(7):501-509.

52. Sharma VK, Lynn A, Kaminski M, Vasudeva R, Howden CW. A study of the prevalence of Helicobacter pylori infection and other markers of upper gastrointestinal tract disease in patients with rosacea. Am J Gastroenterol. 1998;93(2):220-222.
53. Suárez Fernández R, Medina Montalvo S, Trasobares Marugán L, García Rodríguez M. Rosácea y Helicobacter pylori. Estudio de 27 casos [Rosacea and Helicobacter pylori. A study of 27 cases]. Actas Dermosifilogr. 1999;90:162-166. Spanish.

54. Utaş S, Özbakir Ö, Turasan A, Utaş C. Helicobacter pylori eradication treatment reduces the severity of rosacea. J Am Acad Dermatol. 1999;40(3):433-435.

55. Bamford JT, Tilden RL, Blankush JL, Gangeness DE. Effect of treatment of Helicobacter pylori infection on rosacea. Arch Dermatol. 1999;135(6):659-663.

56. Daković Z, Vesić S, Vuković J, Milenković S, Janković-Terzić K, Dukić S, Pavlović MD. Ocular rosacea and treatment of symptomatic Helicobacter pylori infection: a case series. Acta Dermatovenerol Alp Pannonica Adriat. 2007;16(2):83-86.

57. El-Khalawany M, Mahmoud A, Mosbeh AS, A B D Alsalam F, Ghonaim $\mathrm{N}$, Abou-Bakr A. Role of Helicobacter pylori in common rosacea subtypes: a genotypic comparative study of Egyptian patients. J Dermatol. 2012;39(12):989-995.

58. Saleh P, Naghavi-Behzad M, Herizchi H, Mokhtari F, Mirza-AghazadehAttari M, Piri R. Effects of Helicobacter pylori treatment on rosacea: a single-arm clinical trial study. J Dermatol. Epub 2017 April 28.

59. Sato D, Yanaka A, Shibahara T, et al. Peroxiredoxin I protects gastric mucosa from oxidative injury induced by $\mathrm{H}$. pylori infection. $J$ Gastroenterol Hepatol. 2008;23(4):652-659.

60. Ding SZ, Minohara Y, Fan XJ, et al. Helicobacter pylori infection induces oxidative stress and programmed cell death in human gastric epithelial cells. Infect Immun. 2007;75(8):4030-4039.

61. Gürer MA, Erel A, Erbaş D, Cağlar K, Atahan C. The seroprevalence of Helicobacter pylori and nitric oxide in acne rosacea. Int J Dermatol. 2002;41(11):768-770.

62. Schneider MA, Skinner RB, Rosenberg EW, Noah IW, Smith L, Zwarum A. Serological determination of Helicobacter pylori in rosacea patients and controls. Clin Res. 1992;40:831A.

63. Son SW, Kim IH, Oh CH, Kim JG. The response of rosacea to eradication of Helicobacter pylori. Br J Dermatol. 1999;140(5):984-985.

64. Herr H, You CH. Relationship between Helicobacter pylori and_rosacea: it may be a myth. J Korean Med Sci. 2000;15(5):551-554.

65. Bonamigo RR, Leite CS, Wagner M, Bakos L. Rosacea and Helicobacter pylori: interference of systemic antibiotic in the study of possible association. J Eur Acad Dermatol Venereol. 2000;14(5):424-425.

66. Egeberg A, Weinstock LB, Thyssen EP, Gislason GH, Thyssen JP. Rosacea and gastrointestinal disorders: a population-based cohort study. $\mathrm{Br}$ J Dermatol. 2017;176(1):100-106.

67. Pradhan S, Madke B, Kabra P, Singh AL. Anti-inflammatory and immunomodulatory effects of antibiotics and their use in dermatology. Indian J Dermatol. 2016;61(5):469-481.

68. De Francesco V, Bellesia A, Ridola L, Manta R, Zullo A. First-line therapies for Helicobacter pylori eradication: a critical reappraisal of updated guidelines. Ann Gastroenterol. 2017;30(4):373-379.
Clinical, Cosmetic and Investigational Dermatology

\section{Publish your work in this journal}

Clinical, Cosmetic and Investigational Dermatology is an international, peer-reviewed, open access, online journal that focuses on the latest clinical and experimental research in all aspects of skin disease and cosmetic interventions. This journal is included on PubMed. The manuscript management system is completely online

\section{Dovepress}

and includes a very quick and fair peer-review system, which is all easy to use. Visit http://www.dovepress.com/testimonials.php to read real quotes from published authors 